\title{
Aplicação do Adesivo de Fibrina na Sintese da Cúpula Vaginal em Histerectomias
} Abdominal de Coelhas

\author{
Autor: Ari Gonçalves Lima \\ Orientador: Prof. Dr. Murched Omar Taha
}

Dissertação de Mestrado apresentada à Escola Paulista de Medicina da Universidade Federal de São Paulo (UNIFESPEPM), Programa de Pós-graduação em Técnica Operatória e Cirurgia Experimental, em 27/11/2002.

Objetivo: O estudo morfológico da cúpula vaginal submetida à sintese com adesivo de fibrina em histerectomia abdominal de coelha.

Método: Utilizaram-se 20 coelhas distribuídas aleatoriamente em 2 grupos: no grupo A (GA) foi usado o fio de ácido poliglicólico 3-0 nas sínteses das cúpulas vaginais e no grupo $B$ (GB) o adesivo de fibrina (em 2 cúpulas usou-se o fio de sutura para coibir sangramento). Após 6 semanas avaliou-se na cicatriz das cúpulas vaginais através de rádio-vaginografias, vídeo-vaginoscopias e exame macroscópico a existência de granulomas e fístulas, do teste de pressão a resistência tênsil e do exame histológico a quantidade de colágeno e a cavidade abdominal por laparotomia a formação de aderências.

Resultados: O tempo operatório médio no GA foi 22,8 minutos e no GB 24,6 minutos. Visibilizou-se nas radio-vaginografias deiscência de sutura em 1 cúpula vaginal do GA; nas vídeo-vaginoscopias e no exame macroscópico das vaginas, granulomas em 5 cúpulas do GA e em duas do GB (as em que se usou fio de sutura); nas laparotomias, aderências em 5 coelhas do GA e em três do GB. A média de pressão no teste de resistência tênsil no GA foi de $58,5 \mathrm{mmHg}$ e no GB $61,0 \mathrm{mmHg}$. A percentagem da área de fibrose no exame histológico foi de 31,63\% no GA e 29,17\% no GB. Conclusão: Apesar de necessitar de um tempo maior para aplicação, o adesivo mostrou-se eficiente e não desenvolveu granulomas quando usado na sintese da cúpula vaginal em histerectomia de coelhas.

Palavras-chave: Cúpula vaginal. Histerectomia. Adesivo de fibrina. Granulomas.

Dissertação de Mestrado apresentada ao Departamento de Ginecologia e Obstetrícia da Faculdade de Medicina de Ribeirão Preto, da Universidade de São Paulo, Ribeirão Preto/SP, em 9 de setembro de 2002.

Objetivo: determinar o perfil de segurança clinicolaboratorial da terapia com tibolona em pacientes na pós-menopausa portadoras de diabetes mellitus não insulino-dependente (DMNID).

Método: trata-se de um estudo prospectivo, longitudinal, aberto e não controlado com 24 pacientes na pósmenopausa portadoras de DMNID, tratadas com 2,5 $\mathrm{mg} /$ dia de tibolona, pelo período de 180 dias. Foram realizadas avaliações clínica e nutricional com as medidas antropométricas (IMC, RCQ e \% Gordura); dosagens hormonais (FSH, $\mathrm{LH}, \mathrm{T}_{4}$ livre, TSH); bioquímica sangüinea (uréia, creatinina, bilirrubinas, TGO, TGP, glicose e hemoglobina glicosilada); e ultra-sonografia transvaginal (espessura endometrial e volume uterino) pré e pós-tratamento. Na análise estatística foram aplicados o teste não-paramétrico de KolmogorovSmirnov, análise de variância para medidas repetidas e teste " $t$ " pareado para dois períodos, sendo adotado nível de significância de $5 \%$.
Resultados: após 6 meses, todas as pacientes permaneceram em uso da medicação. Como efeitos colaterais, $8,3 \%$ apresentaram cefaléia, 8,3\% mastalgia e 16,6\% sangramento genital. Houve melhora na \% de gordura corporal $(43,15 \pm 6,39$ vs $41,76 \pm 5,20 ; p=0,01)$ e na pressão arterial diastólica $(87,29 \pm 7,36$ vs $78,33 \pm 11,67$; $\mathrm{p}=0,0005)$. Não houve variações significativas na pressão arterial sistólica, freqüência cardiaca, IMC e RCQ, bem como nos parâmetros bioquímicos. Houve diminuição dos sintomas climatéricos $(22,17 \pm 7,15$ vs $3,08 \pm 3,33 ; p=0,0001)$. A avaliação ultra-sonográfica não revelou variações do volume uterino e espessura endometrial.

Conclusão: a utilização de tibolona durante um período de 6 meses, mostrou bom perfil de segurança clínicolaboratorial em pacientes na pós-menopausa portadoras de DMNID.

Palavras-chave: Diabete melito. Tibolona. Menopausa. 